\title{
Chinese Aid Guyana Medical Team Member HAMA Under the COVID-19 Epidemic Correlation Study with TCSQ
}

\section{Jin Zhengxi ( $\nabla 50807853 @ q q . c o m)$}

Nanjing Medical University

\section{Zhang Xiangxin}

Nanjing Medical University

Qiao Zhiming

Nanjing Medical University

\section{Research Article}

Keywords: COVID-19, foreign aid medical treatment, Chinese aid Guyana medical team members, mental health, coping style

Posted Date: June 23rd, 2021

DOI: https://doi.org/10.21203/rs.3.rs-596604/v1

License: (c) (i) This work is licensed under a Creative Commons Attribution 4.0 International License. Read Full License 


\section{Chinese aid Guyana medical team member HAMA under the}

\section{COVID-19 epidemic correlation study with TCSQ}

Jin Zhengxi ${ }^{1}$ Zhang Xiangxin ${ }^{2}$ Qiao Zhiming ${ }^{3}$

1. The first author and corresponding author, Department of Nephrology, The Affiliated Suzhou Hospital of Nanjing Medical University(Suzhou Municipal Hospital),242 Guangji Road, Suzhou City, Jiangsu Province, China.550807853@qq.com

2. Co-corresponding author, Department of Orthopedics, The Affiliated Suzhou Hospital of Nanjing Medical University(Suzhou Municipal Hospital), Email: lovezxx2003@qq.com

3.Department of Surgery, The Affiliated Suzhou Hospital of Nanjing Medical University(Suzhou Municipal Hospital), Email: zhiming_qiao@163.com

\section{[Summary]}

[Purpose]: To understand the mental health level (HAMA) and trait coping style (TCSQ) of the Chinese aid Guyana medical team members (CAGMTM) under the new coronavirus (COVID-19) epidemic, in order to take effective measures to improve the CAGMTM Mental health provides a theoretical basis.

[Method]: From October 2020 to March 2021, the team members have worked and lived in Guyana for 6 months. The mental health level (Hamilton Anxiety Scale, HAMA) and trait coping styles were investigated for 16 team members who aided Guyana.

[Results]: The HAMA score of the CAGMTM was (14.75 \pm 5.12$) ; 14$ doctors had a score of $(15.57+6.01) ; 2$ chefs had a score of $(17.5+7.78)$. The results of multivariate regression analysis showed that among the CAGMTM. Whether there are team members in isolation $(\mathrm{t}=3.383, p<0.05)$, and whether any medical staff in the hospital where the CAGMTM are located have tested positive for COVID-19 ( $\mathrm{t}=-2.831$, $p<0.05)$ are important factors affecting the mental health of the team members during the epidemic. factor. The score of active coping in the coping style is $(29.38 \pm 6.28)$ points, which is lower than the Chinese conventional model $(\mathrm{t}=-0.392, p<0.05)$; the negative coping score is $(30.37 \pm 5.05)$ points, which is higher than the Chinese 
conventional model $(\mathrm{t}=4.908), p<0.05)$; the HAMA score of the CAGMTM in the COVID-19 epidemic was negatively correlated with the positive response in TCSQ $(\mathrm{r}=-0.654, p<0.05)$; the HAMA score was positively correlated with the negative response in TCSQ $(r=0.654), \mathrm{p}<0.05)$.

[Conclusion]: The mental health level of the CAGMTM under the COVID-19 epidemic is closely related to their idiosyncratic coping styles. For foreign aid medical team members working in countries with severe epidemics, attention should be paid to the mental health of foreign aid medical team members, timely intervention should be given, and psychological counseling should be carried out regularly, so that the aid team members can work and live better.

[Keywords] COVID-19; foreign aid medical treatment; Chinese aid Guyana medical team members; mental health; coping style.

The WHO declared the outbreak of COVID-19[1] infection in China as a "Public Health Emergency of International Concern" ${ }^{[2]}$ on January 30, 2020. After China has actively mobilized and organized the people across the country to fight COVID-19 scientifically, dynamically and effectively, the epidemic has been brought under control. However, due to different levels of attention and prevention and control measures in other countries, the COVID-19 pandemic has resulted. In this context, The 16th Chinese Medical Team Aid to Guyana arrived in Guyana on October 1, 2020 for medical assistance. At the same time, it imparted China's COVID-19 prevention and anti-epidemic experience and donated medicine equipment and anti-epidemic materials. Due to the limited capacity of local nucleic acid testing, national nucleic acid testing cannot be performed. Medical staff are at greater risk of contacting COVID-19 patients and infection during diagnosis and treatment, and they are extremely prone to cross-infection and occupational exposure. The infection of COVID-19 among medical staff in the department puts medical team members under tremendous psychological pressure in their daily stressful and arduous work, which will affect their physical and mental health and the quality of clinical work, and even affect their ability to cope with work. This study aims to explore the correlation 
between the mental health level of the aid team members in the COVID-19 epidemic and their idiosyncratic coping styles. Provide theoretical basis for CAGMTM to carry out psychological intervention and improve work quality.

\section{Objects and methods}

\subsection{Survey object}

In March 2021, this study selected 16 members (14 doctors and 2 chefs) of the 16th China-aided Guyana Medical Team for investigation.

\section{Inclusion criteria:}

(1)Selected by the Jiangsu Provincial Office of International Cooperation and Exchange, the doctor has a doctor's qualification certificate, the chef has a cook certificate, and has no criminal record, and the doctor has passed the medical council assessment in Guyana and obtained the local practicing qualification certificate.

(2)No mental illness or major physical illness.

(3)Informed consent to this research, voluntarily join the research project.

\section{Exclusion criteria:}

(1)Severe physical illness;

(2)Past or current mental illness.

A total of 16 CAGMTM were included in this study, with an age distribution ranging from 34 to $49(42.50 \pm 4.44)$ years; education level: 2 junior college students, 14 undergraduates and above; Aid hospital level: 11 people are at national level and 5 people are at the regional level; Isolation of team members: 11 people are isolated, 5 people are not isolated; whether the hospital is admitted to treat COVID-19 patients: yes 11 people, no 5 people; whether the doctor's department has a colleague who has confirmed COVID-19 positive status: Yes 9 people, No 5 people.

\subsection{Survey method}

1.2.1 General information questionnaire items include gender, age, marital status, education level, job title, hospital level, health status, whether there are team members isolated, whether it is a national designated hospital for COVID-19, and whether colleagues in the department have COVID- 19 positive cases.

\subsubsection{Hamilton Anxiety Scale}


Hamilton Anxiety Scale (HAMA) ${ }^{[3]}$ is often used clinically to diagnose and classify anxiety disorders. All items on the scale adopt a 5-level scoring method from 0 to 4 points. The scoring standards at all levels are: 0 points: asymptomatic; 1 point: mild; 2 points: moderate; 3 points: severe; 4 points: extremely severe. The higher the score, the more severe the anxiety symptoms. According to the information provided by the Chinese Scale Cooperation Group ${ }^{[4]}$, The total score of the scale is $\geqslant 29$ points, which may be severe anxiety; the total score $\geqslant 21$ points, there must be obvious anxiety; the total score $\geqslant 14$ points, there must be anxiety; $>7$ points, there may be anxiety; $<7$ points, there is no anxiety symptoms. The Cronbach's $\alpha$ coefficient of the scale is 0.835 , which has good reliability.

\subsubsection{Trait Coping Style Questionnaire}

Trait coping style questionnaire (TCSQ) consists of 20 items, including 10 items for positive response and 10 items for negative response. The questionnaire adopts a five-level scoring method, with scores of 5, 4, 3, 2, and 1 respectively indicating complete agreement, agreement, neutrality, disagreement, and complete disagreement. It is used to reflect the subjects' positive and negative attitudes and behavior characteristics when facing difficulties and frustrations.A high score for active coping reflects that the active coping characteristic is obvious, and a high score for negative coping reflects that the negative coping characteristic is obvious. The scale for positive response to Cronbach's $\alpha$ is 0.89 , and negative response to Cronbach's $\alpha$ is 0.78, which has good reliability.

\subsection{Statistical methods}

SPSS 26.0 statistical software was used for data analysis, general data was described by frequency, quantitative data was described by $(\overline{\mathrm{x}} \pm \mathrm{S})$, and the comparison between the sample and the domestic regular model was constructed by t-test. The correlation between mental health level and trait coping style was Pearson correlation For sex analysis, the test level $\alpha$ is set to 0.05 unless otherwise specified, with a two-sided probability.

\section{Results}

\subsection{HAMA scores}


The total HAMA score of the CAGMTM is $(14.75 \pm 5.12)$ points. Items 1 to 6 and 14 are mental anxiety with a score of $(12.88 \pm 4.06)$; items 7 to 13 are physical anxiety with a score of $(1.88 \pm 2.80)$. 0 were severely anxious, 2 were obviously anxious, scored (25.50 \pm 3.54$)$, and 9 were mildly anxious, scored $(15.11 \pm 1.27)$.

\subsection{Single factor analysis of mental health status scores of CAGMTM}

Whether the CAGMTM are isolated, whether there are colleagues in the department where the COVID-19 is diagnosed and HAMA scores are compared, the difference is statistically significant $(p<0.05)$. The other team members who are quarantined around the team tend to have a higher tendency to score (13.45 \pm 2.91$)$, and the department has a colleague with a positive score of COVID-19 (16.88 \pm 4.67$)$ (Table 1, t-test).

Table 1 Single factor analysis of the scores of mental health status of the CAGMTM

\begin{tabular}{|c|c|c|c|c|}
\hline Project & $\mathbf{n}$ & HAMA & $\mathbf{F}$ & $p$ \\
\hline \multicolumn{5}{|l|}{ Age } \\
\hline $34-40$ & 7 & $16.14 \pm 5.58$ & \multirow{3}{*}{1.368} & \multirow{3}{*}{0.323} \\
\hline & & & & \\
\hline $41-49$ & 9 & $13.67 \pm 4.77$ & & \\
\hline \multicolumn{5}{|l|}{ Hospital level } \\
\hline \multirow[t]{2}{*}{ National level } & 10 & $13.6 \pm 3.03$ & \multirow{3}{*}{7.722} & \multirow{3}{*}{0.0042} \\
\hline & & & & \\
\hline Regional level & 4 & $16.25 \pm 8.42$ & & \\
\hline
\end{tabular}


Education background

Junior college

2

$17.5 \pm 7.78$

Bachelor or above

14

$14.36 \pm 4.92$

2.501

0.1179

Teammate isolated or not?

$\begin{array}{llccc}\text { Yes } & 11 & 13.45 \pm 2.91 & & \\ \text { No } & 5 & 17.6 \pm 7.89 & & 0.0030^{*}\end{array}$

Department colleagues covid positive or not?

Have

No

Note: $* p<0.05$

\subsection{Regression analysis of influencing factors of mental health status of the}

\section{CAGMTM}

A multi-factor linear regression analysis of the mental health status of the CAGMTM, taking the anxiety of the CAGMTM as the dependent variable, and whether the CAGMTM are isolated and whether a colleague in the department is diagnosed with COVID-19 as the independent variables. Regression analysis showed that the difference was statistically significant $(p<0.05)$. The adjusted R-square, $\Delta \mathrm{R}^{2}=0.665$ (Table 2).

Table 2 Regression analysis of HAMA influencing factors of the CAGMTM

\begin{tabular}{cccccccc}
\hline Influencing factors & $\mathrm{B}$ & $\mathrm{SE}$ & $\mathrm{Beta}$ & $\mathrm{t}$ & $p$ & $\mathrm{R}$ square & $\Delta \mathrm{R}$ square \\
\hline $\begin{array}{c}\text { Teammates } \\
\text { isolated or not? }\end{array}$ & 6.286 & 1.858 & 0.549 & $3.383^{*}$ & 0.005 & & 0.665 \\
$\begin{array}{c}\text { Department colleagues } \\
\text { positive or not? }\end{array}$ & -4.914 & 1.736 & -0.460 & $-2.831^{*}$ & 0.014 & & \\
\hline
\end{tabular}

Note: ${ }^{*} \mathrm{p}<0.05, \Delta \mathrm{R}^{2}=0.665$

\subsection{TCSQ scores of the CAGMTM $(\overline{\mathbf{x}} \pm S)$}

The TCSQ scores of the CAGMTM were compared with the Chinese norm. Their positive response $(29.38 \pm 6.28)$ score was lower than the Chinese norm 
(30.26 \pm 8.74$)$; the negative response $(30.37 \pm 5.05)$ score was higher than the Chinese norm ( $21.25 \pm 7.41)$ points, the difference was statistically significant $(p<0.05)$ (Table $3)$.

Table 3 TCQS scores ofthe CAGMTM $(\bar{x} \pm s)$

\begin{tabular}{cccc}
\hline Project & $\mathrm{n}$ & Positive response & Negative response \\
\hline CAGMTM & 16 & $29.38 \pm 6.28$ & $30.37 \pm 5.05$ \\
Domestic norm & 1305 & $30.26 \pm 8.74$ & $21.25 \pm 7.41$ \\
$\mathrm{t}$ & & -0.392 & 4.908 \\
$p$ & & $0.0457^{*}$ & $0.0364^{*}$ \\
\hline
\end{tabular}

Note: ${ }^{*} p<0.05$

\subsection{Pearson's correlation analysis between the total scores of HAMA and the} total scores of coping styles

The HAMA total score of the CAGMTM was significantly correlated with the coping style, the positive coping style was negatively correlated with the anxiety total score $(\mathrm{r}=-0.654, p<0.05)$, and the negative coping style was positively correlated with the anxiety total score $(r=0.654, p<0.05)$, in the face of COVID-19, between the anxiety and coping styles of the CAGMTM, there is a negative correlation between active coping and mental anxiety $(p<0.05)$,Negative coping and mental anxiety were positively correlated, and the difference was statistically significant $(p<0.05)$ (Table 4).

Table 4 Correlation between total scores of the CAGMTM' HAMA and total scores of coping styles

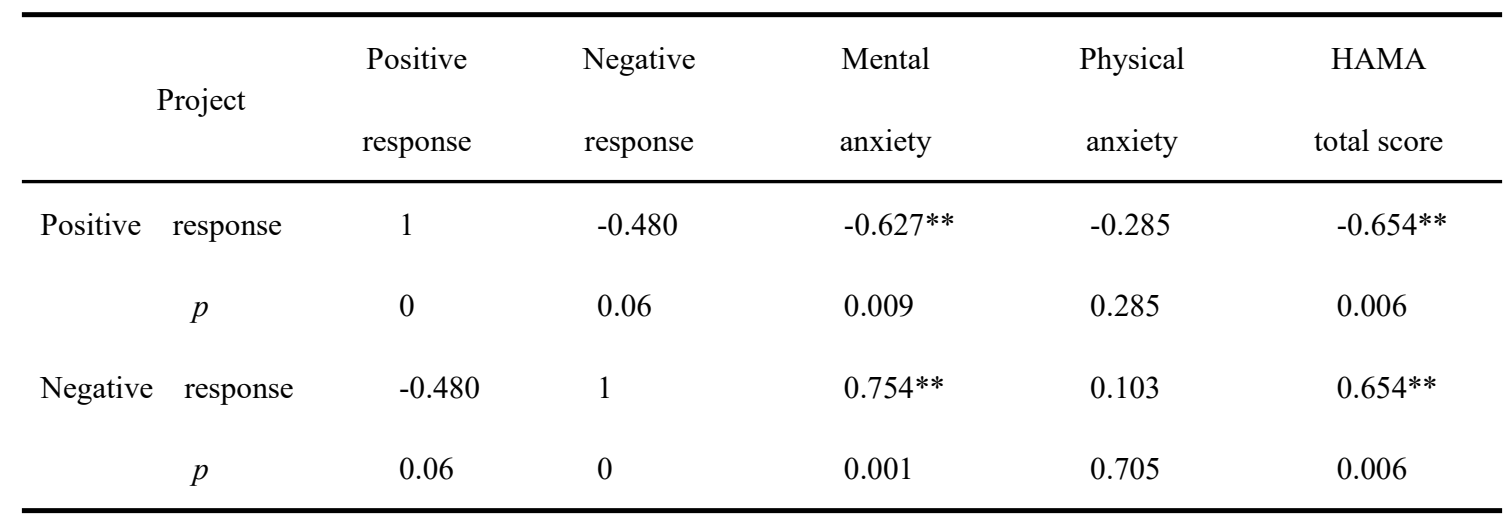




\begin{tabular}{|c|c|c|c|c|c|c|}
\hline \multirow[t]{2}{*}{ Mental } & anxiety & $-0.627 * *$ & $0.754 * *$ & 1 & 0.081 & $0.838 * *$ \\
\hline & $p$ & 0.009 & 0.001 & 0 & 0.767 & 0 \\
\hline \multirow[t]{2}{*}{ Physical } & anxiety & -0.285 & 0.103 & 0.081 & 1 & $0.611^{* *}$ \\
\hline & $p$ & 0.285 & 0.705 & 0.767 & 0 & 0.012 \\
\hline \multirow[t]{2}{*}{ HAMA } & total score & $-0.654 * *$ & $0.654 * *$ & $0.838^{* *}$ & $0.611^{*}$ & 1 \\
\hline & $p$ & 0.006 & 0.006 & 0 & 0.012 & 0 \\
\hline
\end{tabular}

Note: ${ }^{* *} p<0.01,{ }^{*} p<0.05$.

\section{Discussion}

The Cooperative Republic of Guyana is located in the northern part of South America. South America currently has a large number of people infected with COVID-19, and the exchange of people between local residents and surrounding countries affects epidemic control; at the same time, Guyana is one of the countries with the highest immigration rate in the world, with an immigration rate of more than $55 \%$, due to the epidemic, many Guyanese who have immigrated abroad returned to the country to avoid the epidemic, further aggravating the epidemic in their country.

A total of 16 members of the Chinese medical aid team to Guyana, 11 of them assisted the Georgetown Hospital, Guyana's largest national public hospital, and 5 assisted the Linden Hospital, the second largest public hospital in Guyana. Research ${ }^{[5]}$ confirms that most people will have varying degrees of physical and mental stress response when facing work pressure. If they do not adjust in time, they may experience boredom and tiredness at work. Difficulty in exerting subjective initiative, leading to reduced work quality. Different coping styles can affect the development results of things, and even change the emotional state of people, and play an important role in regulating physical and mental health ${ }^{[6]}$.

\subsection{Mental health status of the CAGMTM}

The results of this study showed that the total HAMA score of the CAGMTM was $(14.75 \pm 5.12)$, of which $68.8 \%$ were anxious team members, and those without severe anxiety accounted for $12.5 \%$ of obvious anxiety and $56.3 \%$ of mild anxiety, 
which was higher than Liu Liying ${ }^{[7]}$ research and report the data of foreign aid team members facing the Ebola virus epidemic.

Anxiety is a negative emotional state ${ }^{[8]}$ subjectively felt by an individual, and it is one of the common psychological obstacles faced by medical staff in the face of COVID-19 ${ }^{[9]}$. Each member of the CAGMTM is located in a different department. In addition to contacting patients, they have close contact with local doctors and nurses at work. Nurses take buses to and from get off work. Local buses are also called minibuses. They are small in size and have many passengers. Both increase the probability of potential infection, and also increase the risk of cross-infection and occupational exposure. These risk factors will directly or indirectly affect the physical and mental health of the CAGMTM. Due to the special nature of the work of medical staff, the CAGMTM in the hospital have high risks and heavy responsibilities. They face high-intensity work during the epidemic.Latent anxiety can have a negative psychological impact. In assisting Guyana, team members should strengthen contact and share difficult cases and unsolvable problems encountered in work; teammates discuss and multi-disciplinary consultations to face and solve problems together, and relieve pressure and work in the work. anxiety. In life, the emotional communication between team members should be strengthened, and mutual care should be taken. Help each other, discover and eliminate the bad emotions of teammates in time, and protect the physical and mental health of the team members.

\subsection{The influencing factors of the mental health of the CAGMTM}

The results of multivariate regression analysis showed that whether the CAGMTM are isolated and whether there are COVID-19-positive medical staff in the doctor's department are important factors affecting the mental health of the CAGMTM in the epidemic. The reason for the analysis may be (1) Quarantine teammates increase other teammates' self-infection concerns; (2) The isolation of team members increases teammates' thoughts of their family members and worries about their future work; (3) For team members whose doctors have infections from medical staff in the same department in the hospital, they will consider that the risk of 
infection is significantly increased, and they are afraid of work; (4) The infection of medical staff in the team's department will lead to a shortage of department staff, workload and work pressure; the above reasons will all lead to the psychological pressure of medical team members increased significantly, and negative emotions such as anxiety were extremely easy to appear.

In the special period of the global epidemic of COVID-19, attention should be paid to the mental health of the CAGMTM, coordinated with the hospital to conduct nucleic acid tests in a timely manner, reasonably adjust the diagnosis and treatment shifts, and regularly invite psychologists to perform decompression and psychological counseling, and analyze the reasons for their existence. Look for positive ways to cope, and actively respond to reduce work stress by directly and indirectly affecting negative coping ${ }^{[10]}$. Play an effective intervention for the mental health of the CAGMTM, strengthen the psychological endurance and adaptability, keep a good and positive attitude, and improve the CAGMTM' psychological rescue ability in the face of the outbreak of public health incidents.

\subsection{The situation of the response methods of the CAGMTM}

Coping style is the cognitive or behavioral effort that everyone makes to reduce stress or injury when facing a stressful environment. It is the result of the interaction between individual stability factors and situational factors. The results of this study show that the active response score $(29.38 \pm 6.28)$ of the Chinese aided medical team in the COVID-19 epidemic is lower than the Chinese norm ${ }^{[11]}(30.26 \pm 8.74)$; the passive response score $(30.37 \pm 5.05)$ is higher than that of China Normal mode (21.25 \pm 7.41$)$ points, which are consistent with the results of Zhou Zhenhua ${ }^{[12]}$ in the face of SARS. Research ${ }^{[9]}$ shows that in the COVID-19 epidemic, the length of working hours of clinical front-line staff and the amount of contact with patients during treatment are clearly related to the severity of anxiety.

Under the heavy work and special environment of this investigation, the CAGMTM gradually became anxious with the prolonged working hours of foreign aid, the active response gradually weakened, and the passive coping style gradually increased. The results of Valizadeh ${ }^{[13]}$ showed that negative coping can bring more 
negative emotional experiences, such as pessimism, anxiety, fatigue and so on. This reminds us that in the face of the COVID-19 epidemic, we should strengthen the prevention and control of hospital infections, the rescue of critically ill patients, the learning of professional knowledge and skills, establish a safe and efficient work process, and improve work efficiency under the premise of ensuring the safety of self-protection, and provide rich diagnosis and treatment. Experience and strong professional skills are applied to practice; strengthen teamwork spirit, actively provide psychological counseling, relieve work pressure, increase work enthusiasm, and respond to public health incidents with a positive attitude.

\subsection{Correlation between mental health level and coping styles of the}

\section{CAGMTM}

Coping styles play a mediating role in stressful events and mental health, and there is a correlation with mental health ${ }^{[14]}$. The results of this study showed that the total HAMA score of the CAGMTM was significantly correlated with TCQS, and the positive coping score was negatively correlated with HAMA $(p<0.05)$; the negative coping score was positively correlated with HAMA $(p<0.05)$, and it was positively correlated with Jiang Ganjin ${ }^{[15]}$. The research results are consistent.When the CAGMTM first invested in the recipient country for medical assistance, they were able to actively respond to the COVID-19 epidemic and make self-psychological adjustments, but as time passed, their ability and level to withstand stress events at work decreased, and the department A colleague who tested positive for COVID-19 may make him more sensitive and vulnerable psychologically. The more concerned about the epidemic situation, the more anxious he will be. Worrying about being infected by a patient or colleague, or even worrying about whether he can return home safely or whether he will infect family members through himself, exceeds his normal psychological load, which makes the team members gradually generate anxious negative emotions from the active response.

The CAGMTM have been in a high-intensity and high-pressure environment for a long time, resulting in a decline in their self-regulation ability, which seriously affects work and life. Supportive therapy and stress intervention ${ }^{[15]}$ achieve the 
purpose of treatment by providing methods to eliminate or reduce the negative effects of multiple stress factors. During the period of foreign aid, the CAGMTM should strengthen the systematic training of hospital infection prevention and control knowledge. Do a good job in hospital hand hygiene management, strictly implement the standard prevention system in the diagnosis and treatment process, actively participate in online training and learning organized by domestic experts through the Internet, strictly control the quality of hospital infection management, and reduce the risk of infection for the CAGMTM. The current situation of the COVID-19 epidemic in Guyana is still severe, and the team members should adopt a positive attitude and deal with the pressure correctly. With the help of domestic psychologists, establish a positive psychological response model, improve stress coping skills, improve mental health, reduce anxiety, and enhance psychological adaptability.

In summary, the mental health level and idiosyncratic response methods of the CAGMTM show that in the face of COVID-19 global pandemic stress events, positive psychological interventions should be taken to reduce negative responses and improve the response ability of the CAGMTM to maintain good physical and mental health; establish a complete emergency response plan, comprehensively improve the overall quality of the CAGMTM.

In this study, only 16 team members who aided Guyana in the 16th phase were selected. The sample size is small. When facing public health incidents in foreign aid medical treatment, the mental health status and coping methods of Chinese foreign aid medical team members still need to be further explored.

\section{[Declarations:]}

(1)This study was approved and approved by the Ethical Committee of Suzhou Municipal Hospital.

(2) Ethics approval and consent to participate: The human questionnaire for this study was conducted in accordance with relevant guidelines and regulations. This study was certified by the ethics committee of Suzhou Municipal hospital. All enrolled patients volunteered to participate in the study. Informed consent was obtained from all 
subjects prior to the study.

(3)Consent for publication: All authors agree to the publication of this study.

(4)Availability of data and material: All data and materials in this study belong to the author and consent to be published and used. The supplementary material contains all the original data.

(5)Conflict of Interest: All authors of the article have no conflict of interest.

(6)Funding: This fund belongs to the subject applied by the corresponding author. 2017 Suzhou Key Industrial Technology Innovation Project (People's Livelihood Science and Technology -- Basic Research of Medical and Health Application) ( SYSD2017112).

(7) Author's contribution statement: This research is designed by Jin Zhengxi and Zhang Xiangxin, research implementation and data collection are Qiao Zhiming, Zhang Xiangxin, Jin Zhengxi, and the paper is written by Jin Zhengxi.

(8)Acknowledgements: This article was written with the help of two doctors (Wu Zhengyan and Yang Xuna) from Suzhou Guangji Hospital (Suzhou Mental Health Center). After the article was written, they reviewed the article and would like to send my sincere thanks.

\section{[references]:}

[1]A rapid advice guideline for the diagnosis and treatment of 2019 novel coronavirus (2019-nCoV) infected pneumonia(standard version)[J]. Med J Chin PLA, 2020,45(1):1-20. [DOI:10.11855/j.issn.0577-7402.2020.01.01]

[2]Eurosurveillance Editorial Team. Note from the editors:World Health Organization declares novel coronavirus(2019-nCoV)sixth public health emergency of international concern [J].Euro Surveill,2020,25(5):200131.

DOI:10.2807/1560-7917.ES.2020.25.5.200131e

[3]Hamilton M. The assessment of anxiety states by rating[J]. Br J Med Psychol, 1959,32(1):50-55. DOI:10.1111/j.2044-8341.1959.tb00467.x

[4]Zhang Haihua, Cao Qiaoling.Effect of extended nursing service model on coping style and quality of life of patients with depression[J]. Electronic Journal of Clinical 
Medical Literature,2016,3(9):1679-1682. [DOI: 10.16281/j.cnki.jocml.2016.09.071] [5]Qin H Y, Jia P, Liu H. Nursing strategies for patients with chronic renal failure undergoing maintenance hemodialysis treatment by arteriovenous fistula $[\mathrm{J}]$. Iran $\mathrm{J}$ Public Health, 2016,45(10):1270-1275.

[6]Xu Mingjin, Huang Xiani, Feng Zhiyuan, et al.The mediating effect of coping style on the relationship between work stress and mental health of nuclear enterprise employees[J]. Journal of Environmental and Occupational Medicine, 2016,33 (2):134-138. [DOI: 10.13213/j.cnki.jeom.2016.15278]

[7]Liu Liying, Zhang Xin, Zhang Xiu, et al. Correlation of mental health level and trait coping styles in the frontline medical staff fighting for Ebola virus[J]. Modern Nursing, 2014,20(35):4411-4413.

[8]Gao YQ, Pan BC, Sun W, et al . Anxiety symptoms among Chinese nurses and the associated factors:a cross sectional study[J] . BMC Psychiatry,2012(1)2:141.

DOI:10.1186/1471-244X-12-141

[9]Ruilin Li,Youlin Chen, et al. Anxiety and related factors in frontline clinical nurses fighting COVID-19 in Wuhan[J]. Medicine,2020,99:30.

DOI:10.1097/MD.0000000000021413

[10]Zhong Xia,JING Qianjin,Qin Liju, et al. Correlation Between Stress Reaction and Social Support, Life Events,Coping Style in M edical Personnel[J]. Chinese Journal of Clinical Psychology, 2005,13(1):70-72.

[11] Wang Xiangdong, Wang Xilin, Ma Hong, et al.Manual of mental health rating scales[M]. Beijing: Chinese Journal of Mental Health,1999:122-124.

[12]Zhou Zhenhua, Li Xuemei, Chen Kangning, et al.Relationship between mental health and coping style of first-line medical staff with SARS[J]. Chinese hehavioral medical science,2004(3):305.

[13] Valizadeh L,Zamanzadeh V,Habibzadeh H,et al.Coping strategies to hinder intention to leave in Iranian nurses:a qualitative content analysis[J].Int J Community Based Nurs Midwifery,2015,3 (4):318-327.

[14]Yang Xiaofang,Hu Shaoyun.Related Study between Social Support and Coping Style,and Anxiety and Depression of Nurse[J]. China Journal of Health Psychology, 
2011,19(3):300-301.

[15]Jiang Ganjin, Zhu Yihong et al.Correlation analysis of psychosocial emergency factors and several psychosomatic health indicators[J].Chinese hehavioral medical science,1996,5(4):200-202. 